\title{
Experimental investigation of two-phase gas- liquid flow in microchannel with T-junction
}

\author{
German Bartkus ${ }^{1,2^{*}}$, Igor Kozulin ${ }^{1,2}$, Vladimir Kuznetsov ${ }^{1,2}$ \\ ${ }^{1}$ SB RAS, Kutateladze Institute of Thermophysics, 630090 Ac. Lavrentieva ave. 1, Novosibirsk, \\ Russia \\ ${ }^{2}$ Novosibirsk State University, 630090 st. Pirogova 1, Novosibirsk, Russia
}

\begin{abstract}
Using high-speed video recording and the method of dual laser scanning the gas-liquid flow was investigated in rectangular microchannels with an aspect ratio of 2.35 and 1.26. Experiments were carried out for the vertical flow of ethanol-nitrogen mixture in a microchannel with a cross section of $553 \times 235 \mu \mathrm{m}$ and for the horizontal flow of water-nitrogen mixture in a microchannel with a cross section of $315 \times 250 \mu \mathrm{m}$. The Tmixer was used at the channel's inlet for gas-liquid flow formation. It was observed that elongated bubble, transition, and annular flows are the main regimes for a microchannel with a hydraulic diameter substantially less than the capillary constant. Using laser scanning, the maps of flow regimes for ethanol-nitrogen and water-nitrogen mixtures were obtained and discussed.
\end{abstract}

\section{Introduction}

Multiphase microsystems based on microchannels are perspective for a variety of applications. Typical dimensions of the channels in these microsystems are in the range from hundreds of nanometers to hundreds of micrometers, which ensures the excellent heat and mass transfer properties. Research of gas-liquid flows in microchannels show that it is possible to distinguish 5 basic modes: isolated bubbles, the Taylor bubbles, slug-annular flow, annular and dispersion flow, which properties have been well studied $[1,2]$. Important parameters for optimizing the heat and mass transfer processes in microchannels are the bubble length, the liquid film thickness, and their distribution. In the paper [3], the modes of gas-liquid flow in a microchannel with a gap of $300 \mu \mathrm{m}$ and width from 10 to 30 $\mathrm{mm}$ were presented. Laser scanning method was used in $[4,5]$ for determination of the statistical characteristics of the gas-liquid flows.

The objective of the present work is experimental studying the mechanism of multiscale upward vertical and horizontal gas-liquid flow formation in rectangular microchannels of the microfluidic devices. Using voltage signals of flow from the photodiodes, flow types were defined in microchannels with cross sections of $553 \times 235 \mu \mathrm{m}$ and $315 \times 250 \mu \mathrm{m}$ by dual laser scanning. Deionized water, ethanol, and nitrogen were used as working fluids.

*Corresponding author: germanbartkus@gmail.com 


\section{Experimental equipment and methods}

The experimental setup for studying the upward vertical and horizontal gas-liquid flow in a rectangular microchannel is shown in Figure 1 (a). Nitrogen, which is fed from a tank (5) via the control valve and gas flow meter (2) to the test section, is used as working gas. Water flows from the tank (4) through a flow meter (3) and further into the T-shaped mixer (1) at the inlet of the experimental section. Experimental section is a microchannel, assembled from glued glass and aluminum plates with length $\mathrm{L}=30 \mathrm{~cm}$. The hydraulic diameter is much smaller than the capillary constant. From the exit of experimental section, the gas-liquid mixture is moved to the liquid tank (10), where the gas escapes to the atmosphere.

The study of gas-liquid flow regimes was conducted using the method of dual laser scanning, see Figure 1 (b). Two lasers (7) are positioned so that the laser beams illuminate one side of the rectangular channel. A lens (8) is used to change the diameter of laser spot; an aperture is used to reduce the diameter of the laser beam. The lasers are powered using voltage power source. The intensity of transmitted light is measured with photodiodes (6) arranged at the opposite side of the channel. The electric voltage from the photodiodes is analyzed using high-speed ADC and processed on the computer over the period from one to three minutes. Flow patterns are also registered using the high-speed camera (9). The first laser is located at a distance of $8.5 \mathrm{~cm}$ from the inlet, camera $-19 \mathrm{~cm}$ from the inlet, second laser $-24 \mathrm{~cm}$ for observing the stable gas-liquid flow.

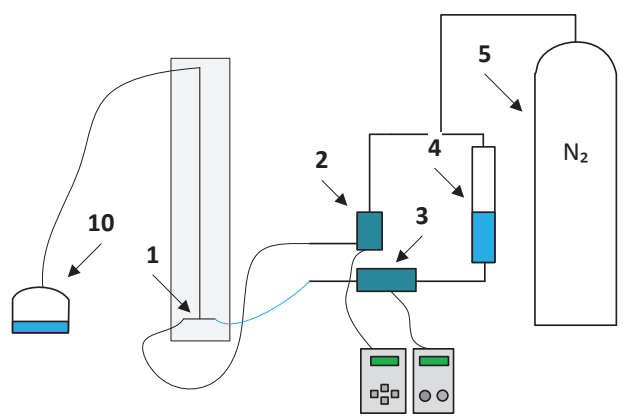

a

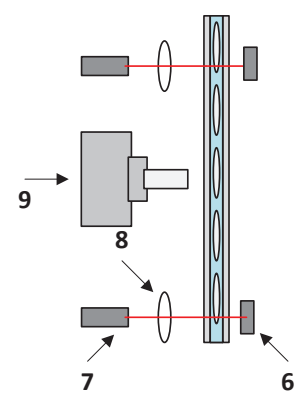

b

Fig. 1. Experimental setup (a), scheme of the dual laser scanning method (b).

\section{Two-phase gas-liquid flow in microchannels}

The wide range of phenomena, which are not typical on a large scale and are poorly understood, arises by the reason of reducing typical size of the flow in microchannel systems. The high-speed video shows that the periodic flow with elongated gas bubbles (Taylor bubbles), the transition flow and the annular flow are the main flow regimes for microchannels with the gap substantially less than the capillary constant. In the microchannel with a cross section of $553 \times 235 \mu \mathrm{m}$ and of $315 \times 250 \mu \mathrm{m}$, the flow with elongated bubbles (slug), the transition flow and the annular one are presented. The slug flow is characterized by the periodicity of the voltage signal from the photodiode. Increasing the superficial gas velocity above the specified values leads to the destruction of the elongated bubbles, and the flow loses its periodicity.

The elongated-bubble flow and the transition flow are presented in Fig. 2 for an upward ethanol-nitrogen experiment in a microchannel $553 \times 235 \mu \mathrm{m}$. The elongated bubble flow is observed in the range of superficial gas velocities $\mathrm{J}_{\text {gas }}=0.126-0.772 \mathrm{~m} / \mathrm{s}$. A fragment of the 
signal from the photodiode for the present flow is shown in Fig. 2 (a). The highest amplitude level from photodiode corresponds to a full filling cross section of the channel by liquid (liquid bridge), the minimum signal corresponds to gas bubble. The visualization of the flow is presented in Fig. 2 (b). The elongated bubble flow is characterized by the periodicity of gas bubbles and liquid bridges.

The transition flow is observed in the range of superficial gas velocities $J_{\text {gas }}=0.617$ $3.012 \mathrm{~m} / \mathrm{s}$. The definition of the boundary from the transition to annular flow is required experiments with higher superficial gas velocities. A fragment of the signal from the photodiode for transition flow is shown in Fig. 2 (c) The visualization of the flow is presented in Fig. 2 (d). Such flow is unstable; liquid bridges appear by combining waves of disturbances on short opposite sides of the microchannel and they are destroyed by combining of gas bubble-slugs.

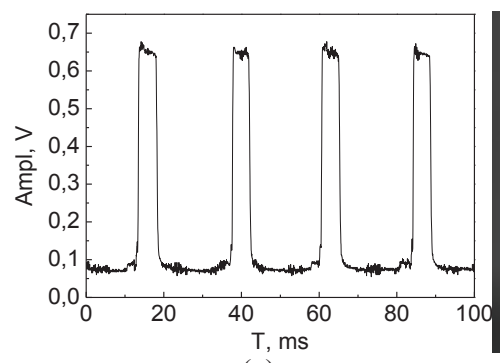

(a)

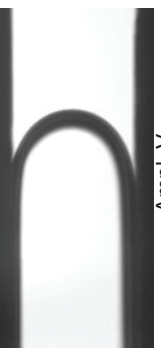

(b)

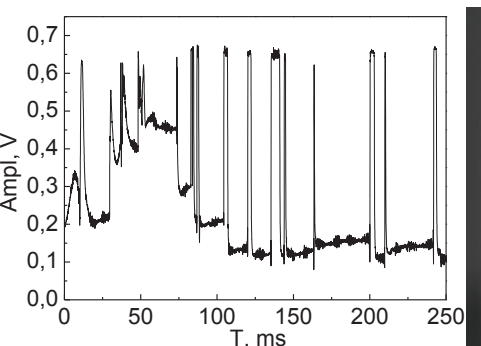

(c)

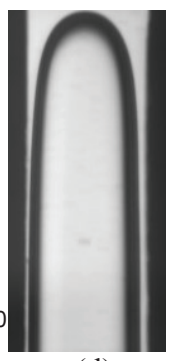

(d)

Fig. 2. Vertical flow in microchannel $553 \times 235 \mu \mathrm{m}$ (ethanol-nitrogen). The dynamics of voltage on the photodiode for elongated-bubble flow at superficial velocities of liquid and gas: $\mathrm{J}_{\mathrm{liq}}=0.042 \mathrm{~m} / \mathrm{s}$, $\mathrm{J}_{\mathrm{gas}}=0.369 \mathrm{~m} / \mathrm{s}$ (a), visualization (b), the dynamics of voltage on the photodiode for transition flow at velocities of liquid and gas: $\mathrm{J}_{\text {liq }}=0.042 \mathrm{~m} / \mathrm{s}, \mathrm{J}_{\mathrm{gas}}=1.814 \mathrm{~m} / \mathrm{s}$ (c), visualization (d).

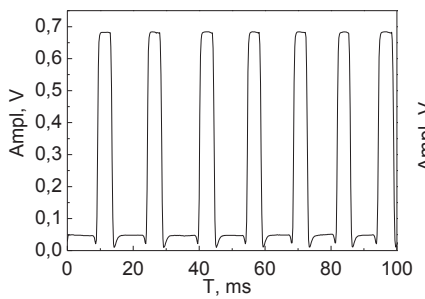

(a)

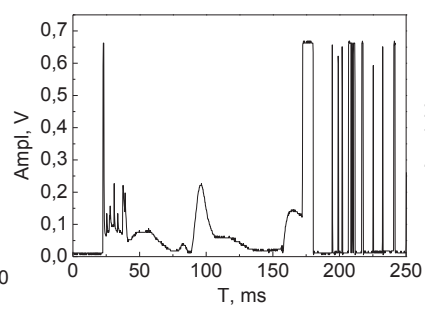

(b)

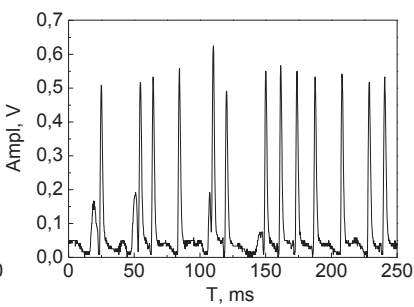

(c)

Fig. 3. Horizontal flow in microchannel of $315 \times 250 \mu \mathrm{m}$ (water-nitrogen). The dynamics of voltage on the photodiode for elongated-bubble flow at superficial velocities of liquid and gas: $J_{\mathrm{liq}}=0.035 \mathrm{~m} / \mathrm{s}$, $\mathrm{J}_{\mathrm{gas}}=0.14 \mathrm{~m} / \mathrm{s}$ (a), for transition flow at velocities of liquid and gas: $\mathrm{J}_{\mathrm{liq}}=0.035 \mathrm{~m} / \mathrm{s}, \mathrm{J}_{\mathrm{gas}}=3.73 \mathrm{~m} / \mathrm{s}$ (b), for annular flow at velocities of liquid and gas: $\mathrm{J}_{\mathrm{liq}}=0.035 \mathrm{~m} / \mathrm{s}, \mathrm{J}_{\mathrm{gas}}=7.26 \mathrm{~m} / \mathrm{s}(\mathrm{b})$.

The elongated-bubble flow and the transition flow are presented in Fig. 3 for the waternitrogen experiment in a horizontal microchannel $315 \times 250 \mu \mathrm{m}$. The elongated bubble flow is observed in the range of superficial gas velocities $J_{\text {gas }}=0.074-0.786 \mathrm{~m} / \mathrm{s}$. A fragment of the signal from the photodiode for the transition flow is shown in Fig. 3 (a).

The transition flow is observed in the range of gas velocities $J_{\text {gas }}=0.641-4.232 \mathrm{~m} / \mathrm{s}$. A fragment of the signal from the photodiode for the transition flow is shown in Fig. 3 (b).

The annular flow is observed in the range of gas velocities $J_{\text {gas }}=4.67-21.056 \mathrm{~m} / \mathrm{s}$. A fragment of the signal from the photodiode for the transition flow is shown in Fig. 3 (c). The highest amplitude level from photodiode does not correspond to a full filling cross section of the channel by liquid (liquid bridge); waves of liquid are observed on short opposite sides of the microchannel. 


\section{Flow pattern maps}

Experiments for horizontal water-nitrogen and vertical upward ethanol-nitrogen types' flows were carried out. Maps were obtained using visualization and signals from photodiodes. 3 regimes for a microchannel with hydraulic diameters $\mathrm{D}_{\mathrm{h}}=279 \mu \mathrm{m}$ $(315 \times 250 \mu \mathrm{m})$ and 2 regimes for a microchannel with hydraulic diameter $D_{h}=326 \mu \mathrm{m}$ were observed. As you can see for the ethanol-nitrogen experiment and water-nitrogen experiment, the boundaries for beginning transition flow are not very different.

For the water-nitrogen experiment, flow pattern map confirms the data obtained by Zhao [6] for the channel with hydraulic diameter $\mathrm{D}_{\mathrm{h}}=400 \mu \mathrm{m}$. In Fig. 4 (b), filled area (1) corresponds to unstable slug (transition flow), filled area (2) corresponds to slug-annular flow.

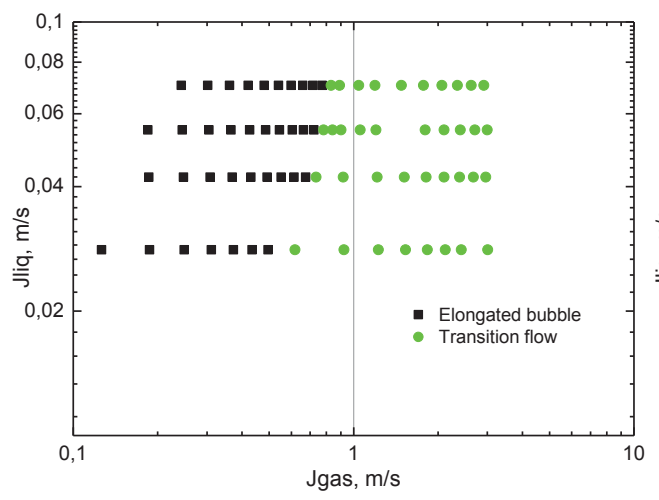

(a)

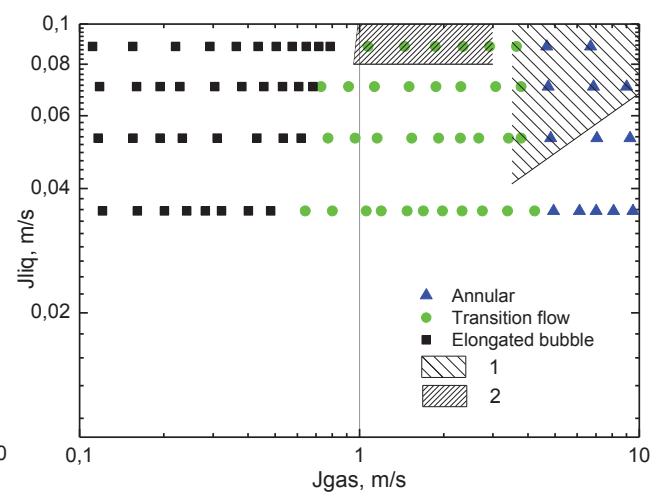

(b)

Fig. 4. Maps of regimes for ethanol-nitrogen mixture in microchannel with hydraulic diameter $\mathrm{D}_{\mathrm{h}}=326 \mu \mathrm{m}(\mathrm{a})$, and for water-nitrogen mixture in microchannel with hydraulic diameter $\mathrm{D}_{\mathrm{h}}=279 \mu \mathrm{m}$ (b).

\section{References}

Laser scanning method and high-speed visualization are an informative technique to obtain the data of two-phase flow. It was observed that the regimes with elongated gas bubbles, the transition flow, and annular flow are the main patterns for a microchannel with a hydraulic diameter substantially less than the capillary constant. Maps of flow regimes in a rectangular microchannel with hydraulic diameter $D_{h}=326 \mu \mathrm{m}(553 \times 235 \mu \mathrm{m})$ for the ethanol-nitrogen mixture, $\mathrm{D}_{\mathrm{h}}=279 \mu \mathrm{m}(315 \times 250 \mu \mathrm{m})$ for water-nitrogen were obtained and are shown in Fig. 4.

This work performed in the Kutateladze Institute of Thermophysics SB RAS by a grant from the Russian Science Foundation (project No 16-19-10519).

\section{References}

1. J.W. Coleman, S. Garimella, Int. J. Heat Mass Trans. 42 (1999)

2. F. A. Kawahara, A., P.M.Y. Chung, M. Kawaji, Int. J. Multiphase Flow 28 (2002)

3. E. A. Chinnov, V. Ronshin, O. A. Kabov, Thermophys. Aeromech. 21 (2015)

4. R. Revellin, J. R. Thome, J. Micromech. Microeng. 17 (2007)

5. I.A. Kozulin, V.V. Kuznetsov, J. Appl. Mech. Tech. Phys. 52 (2011)

6. Yuchao Zhao, Guangwen Chen, Chunbo Ye, Quan Yuan, Chem. Eng. Sci. 87 (2013) 